\title{
Study on Dynamics of Calculus Nonlinear Oscillator
}

\author{
Yong Huang ${ }^{1,}$ Zefu zhao ${ }^{1,}$ Yanchun Zhao ${ }^{1}$ \\ ${ }^{1}$ College of Mathematics and Statistics, Zhaotong University, Zhaotong, Yunnan, 657000 \\ a email
}

Keywords: Dynamics, Calculus, Nonlinear Oscillator

\begin{abstract}
The fractional derivative can be regarded as the Volterra integral of the Abel kernel function and its value is not only closely related to the value of the current moment, but also to the whole history. Therefore, the application of fractional calculus to some viscoelastic materials can well describe the time effect of materials, such as many polymer materials. Compared with the classical viscoelastic constitutive model, the fractional differential viscoelastic constitutive model can not only describe the constitutive relation and mechanical properties of viscoelastic materials, but also can be used in a wide frequency range to describe the mechanical behavior of the material.
\end{abstract}

\section{Introduction}

One of the keys to study the dissipative structure and its mechanical properties is to establish the viscoelastic constitutive relationship of the material accurately, so that it can describe the actual mechanical properties of the material well. The classical viscoelastic constitutive model is very limited in describing the constitutive relationship and mechanical properties of viscoelastic materials. A lot of experiments and engineering practice have shown that at least for a large class of polymer materials, fractional differential Type viscoelastic constitutive relationship can describe the mechanical behavior of the material in a wide frequency range, and can easily consider the temperature factor in the model, and the model is simple, the parameters needed to determine the model is relatively easy to determine by experiment and so on. It is a more accurate model of viscoelastic constitutive model. In particular, recent studies have shown that fractional calculus has a close relationship with fractal dynamics.

So far, most researches on the nonlinear vibration system of viscoelastic structures are based on the viscoelastic constitutive relation and mechanical behavior described by integers and integral operators, while the nonlinear constitutive relations based on fractional differential type The dynamics of the system is rarely found in the literature. In this paper, we study a set of nonlinear dynamical systems with nonlinear fractional derivative, establish a numerical solution scheme, and analyze its nonlinear dynamic characteristics.

\section{Development Status of Nonlinear Vibration}

The purpose of nonlinear vibration theory is to determine the qualitative characteristics and quantitative laws of the system motion under different parameters and initial conditions based on the mathematical model of nonlinear vibration system. Nonlinear vibration systems are described by nonlinear differential equations. Because of the nonlinear term, the superposition principle can not be established. Therefore, it is much more complicated to study the nonlinear vibration than to study the linear vibration. For the nonlinear differential, except for a few can find accurate analytic solution for a long time, there is no suitable for all types of equations of the common precise analytic solution, it is difficult to get accurate analytic solution, for practical engineering nonlinear problems, in addition to In addition to the experimental method, the commonly used theoretical methods are geometric method, analytic method and numerical method.

The geometric method is a qualitative method to study nonlinear vibration. The traditional geometric method is to use the phase plane of the phase trajectory as a visual description of the movement process. On the basis of qualitative theory of ordinary differential equations, the properties of solutions of differential equations are determined according to the geometric 
properties of phase trajectories. The singular points and limit cycles in the phase plane are used as the geometric representation of the equilibrium state and the isolated period. The geometrical method not only can obtain the intuitionistic qualitative result, moreover may provide the theory basis for other research methods.

The analytic method is a quantitative method to study the motion of nonlinear system. That is, by accurately or nearly seeking the analytical solution of nonlinear differential equations, the nonlinear vibration of the law of motion, as well as the system parameters and initial conditions of dependence. The exact solutions of nonlinear differential equations usually involve the introduction and study of non-elementary functions. The analytical method not only can determine the law of the nonlinear system motion with time, but also can get the dependency relation between the motion characteristic and the system parameter, so it is an important method of the nonlinear vibration research. The numerical method is to study the numerical method of nonlinear vibration system. The numerical method is used to solve the nonlinear differential equations by numerical method, and the laws of motion of the nonlinear system under the conditions of specific parameters and initial conditions are obtained. Numerical methods can be used to calculate the time histories of various movements of a particular nonlinear system. The influence of the parameters on the motion of the system can also be determined numerically, and the influence of the initial conditions on the motion of the system can be determined by calculation of the basin and its boundary. But numerical methods can only be carried out with limited accuracy. In the possible conditions, must be combined with theoretical and experimental studies to test.

\section{Nonlinear Fractional Operator}

In recent years, nonlinear fractional differential viscoelastic constitutive relations have been paid much attention by researchers. The results show that a large class of polymeric damping materials exhibit nonlinear mechanical properties under large strain and can not be modeled accurately by linear fractional differential viscoelastic constitutive model. It is also found that the nonlinearity of some polymeric damping materials. The viscoelastic mechanical properties can be accurately described by the nonlinear fractional differential constitutive model. YMeshaka, EMainardi, MikaelEnelund and so also studied the nonlinear fractional differential physical nature, numerical solution and other issues.

Fractional operators commonly used in the process can be considered as Volterra integral operations on Abel kernel functions. The complexity and difficulty of the numerical algorithm are not only related to the nonlinear properties of the unknown function, but also to the singularity of the Abel kernel function of the fractional operator. While the analytical solution can not be obtained, the numerical solutions of some kinds of nonlinear oscillator differential equations with fractional operators are deduced. The singularity problem in the definition of Riemann-Liouville fractional derivative is established by Zhang-Shimizu method and Newmark method, and then the singular value method of nonlinear fractional calculus is obtained.

\section{Numerical Study of Duffing Oscillator with Fractional Differential Type}

As a classical nonlinear oscillator, Duffing equation characteristics are extensive and in-depth study. It has a wealth of dynamic behavior, these actions are still the focus of people's research. Cao Qingjie studied the static and global dynamic bifurcation of the Duffing equation. By studying the global behavior of the mean equation, the conditions of various bifurcations were obtained, and the change of the periodic solution of Duffing equation and its nonlinear properties were revealed. We often discuss the Duffing oscillator will be studied with the system parameters change chaotic vibration of the process, that is, the way to produce chaotic vibration. It is helpful to deepen the understanding of the process of chaotic vibration and to clarify the mechanism of the emergence of chaotic vibration. In practice, it is found that the way to generate chaos is also an effective method to distinguish chaotic vibration, especially chaotic vibration and random vibration. For a system with irregular aperiodic motion, if the chaos is presented as the parameter changes, the system can 
be chaotic rather than random.

(1) The numerical algorithm of fractional differential type Duffing oscillator equation has high precision, fast convergence and good stability. The correctness, reliability and reliability of the numerical algorithm developed in this paper are further proved by calculating the classical Duffing equation.

(2) When the fractional derivative order of the fractional differential type Duffing oscillator is small, the oscillator enters the chaos by doubling period vibration;

(3) Different steady-state Duffing oscillator with different frequency can produce different stable periodic motions. When the excitation frequency is small, the vibrator is a double attractor-type chaotic vibration.

(4) The fractional differential type Duffing oscillator is more likely to enter chaos than the classical Duffing oscillator under the same structural parameters and initial conditions, with the same excitation intensity. The smaller the fractional derivative order is, the smaller the critical excitation of the oscillator enters chaos.

\section{Fractional Vanderpol Oscillator Algorithm and Its Nonlinearity}

Classical vanderPol system as the most typical non-linear dynamic system, is due to the system caused by nonlinear damping, early on its research is mainly in the tube oscillation and simulation of human heart beat based on the classic forced The vanderpol system represents a typical unorthodox form of oscillations. The nderpol equation describes such oscillatory oscillations as sine oscillations as relaxation oscillations and simulates the oscillation of the heart. The nderpol equation also represents various oscillatory circuits with negative resistance elements, Single junction and tunnel diodes and other components have negative resistance. The analytical method of the classical vanderpol system is the nonresonance, the primary resonance, the superharmonic resonance, the subharmonic resonance and the combination of the two harmonic vibrations under simple harmonic excitation. Resonance and its periodic solution and frequency characteristics show that the nonlinear system is strongly nonlinear.

A numerical algorithm for the fractional differential vanderpol oscillator with Riemann-uouvine fractional derivative is derived by combining the numerical algorithm of nonlinear fractional differential oscillators with the Newmark method. An example shows that the algorithm is stable and has high precision, fast convergence and easy to improve to further improve the accuracy.

Fractional differential type Longde: Pol oscillator's fractional differential damping term makes the oscillator in the self-excited vibration into the steady period of time after the slow movement.

The vibrator is chaotic when the fractional derivative is small under forced vibration;

The excitation frequency increases and the period number of the periodic vibration decreases, and the double attracting ring appears in the excitation frequency increasing process.

\section{Numerical Study of a Type Fractional Order Nonlinear Oscillator}

Fractional differential viscoelastic constitutive equations are studied extensively and extensively. A lot of experiments show that the viscoelastic constitutive relation of fractional differential type can accurately describe the mechanical behavior of some polymer damping materials, and has unique characteristics. Fractional differential viscoelastic constitutive relations have attracted the attention of researchers. Many scholars have introduced fractional micro-integral theory into nonlinear functions, and obtained nonlinear fractional-differential viscoelastic constitutive equations. Such as N.Shimizu's group with a large number of experimental data show that the quadratic function of the fractional differential constitutive relationship can well describe the high-performance impact damping material constitutive relations: Zhang Wei, Sun Haizhong experimental study also shows that Limitations of Viscoelastic Constitutive Relation of Linear Fractional Differential Type for Mechanical Behavior of Polymer Materials under Large Deformation.

The nonlinear term has less influence on the dynamic behavior of the system than the linear term; 
The nonlinear coefficient increases, the dissipation of the oscillator system increases, the vibration amplitude decreases;

In the case of linear damping, the higher the fractional derivative order, the faster the oscillator decay. Forced vibration:

The order of the fractional derivative increases, and the super-resonant response of the vibrator increases;

The nonlinear coefficient increases and the super - resonant response of the vibrator increases.

The linear coefficient increases and the super - resonant response of the vibrator decreases.

The higher the external excitation force is, the stronger the super-resonant response is, and the vibration leads to chaos.

\section{Conclusion}

In this paper, we study the mechanical properties of several kinds of fractional differential type nonlinear oscillators, which can be regarded as a nonlinear dissipative system with the introduction of polymer damping in the classical nonlinear system. The study of the mechanical behavior of this type of nonlinear dissipative system has important theoretical and practical significance for exploring the physical meaning of fractional calculus, the relationship between fractional calculus and fractal, and solving various non-linear behaviors of dissipative structures.

In this paper, a numerical method is proposed to solve the problem of fractional differential type nonlinear oscillator differential equation, which can effectively solve the problem of quadratic nonlinear fractional derivative and cubic nonlinear fractional derivative when the analytic solution can not be obtained. This numerical method combines the Shimizu method and the Newmark method to solve the singularity problem in the definition of Riemann-Liouville fractional derivative, and obtains the one-step numerical product scheme for the solution of nonlinear fractional calculus. The numerical examples show that the proposed algorithm has high accuracy, good stability, fast convergence speed and high efficiency.

\section{Acknowledgements}

Project Title: Research Projects Supported by the Scientific Research Fund of Yunnan Department of Education

Project Number: 2014Y499

\section{References}

[1] Zhu Zhengyou, Li Genguo, Cheng Changjun. Static and Dynamic Behavior of Viscoelastic Timoshenko Beam with Fractional Derivative Constitutive Relation [J]. Journal of Applied Mathematics and Mechanics, 2006 (01)

[2] Cao Qingjie, Zhang Tiande, Li Jiuping. Study on static and dynamic bifurcation of Duffing equation [J]. Application Mathematics, 2007 (02)

[3] Yang Tingqing. Study on creep rupture theory of cracked body and its application [J]. Advances in Mechanics, 1999 (02)

[4] Huang Junqi, He Guangyu, Liu Ciqun .Analysis of Generalized Second-Order Fluid Motion in a Binocular Rheometer [J]. Science in China Series A Mathematical Physics Journal of Astronomy and Technology, 2006 (10)

[5] Chai Jian. Dynamic characteristics of viscoelastic damper [J]. Journal of Gansu University of Technology, 1995 (01) 